\title{
On some new sequence spaces
}

\author{
Ekrem SAVAŞ* \\ Department of Mathematics, Uşak University, Uşak, Turkey \\ Geliş Tarihi (Recived Date): 04.11.2018 \\ Kabul Tarihi (Accepted Date): 25.11.2018
}

\begin{abstract}
In this paper, we investigate some new sequence spaces which arise from the notation of generalized de la Vallée-Poussin means and introduce the spaces of strongly $\lambda$-invariant summable sequences which happen to be complete paranormed spaces under certain conditions.
\end{abstract}

Keywords: $\sigma$ - convergence, absolutely lambda- invariant, strongly lambda invariant summability.

\section{Bazı yeni dizi uzayları üzerine}

\section{Özet}

Bu makalede, genelleştirilmiş de la Vallée-Poussin ortalamalarından ortaya çıkan bazı yeni dizi uzaylar incelenmiş ve belirli koşullar altında tam paranormlu uzay olan kuvvetli $\lambda$-değişmez toplanabilir dizi uzayları tanıtılmıştır.

Anahtar kelimeler: $\sigma$ - yakınsama, mutlak lambda- değişmez, güçlü lambda değişmez toplanabilirlik.

\section{Introduction}

Let $w$ be the set of all sequences real or complex and $\ell_{\infty}$ denote the Banach space of bounded sequences $x=\left\{x_{k}\right\}_{k=0}^{\infty}$ normed by $\|x\|=\sup _{k \geq 0}\left|x_{k}\right|$. Let $D$ be the shift operator on

\footnotetext{
*Ekrem SAVAŞ, ekremsavas@yahoo.com
} 
$w$, that is, $D x=\left\{x_{k}\right\}_{k=1}^{\infty}, D^{2} x=\left\{x_{k}\right\}_{k=2}^{\infty}$ and so on. It may be recalled that [see Banach [1]] Banach limit $L$ is a nonnegative linear functional on $\ell_{\infty}$ such that $L$ is invariant under the shift operator (that is, $L(D x)=L(x) \forall x \in \ell_{\infty}$ ) and that $L(e)=1$ where $e=\{1,1, \ldots\}$. A sequence $x \in \ell_{\infty}$ is called almost convergent (see, [5]), if all Banach limits of $x$ coincide. Let $\hat{c}$ denote the set of all almost convergent sequences. Lorentz [5] proved that

$\hat{c}=\left\{x: \lim _{m \rightarrow \infty} \frac{1}{m+1} \sum_{i=0}^{m} x_{n+i}\right.$ exists uniformly in $\left.n\right\}$.

Several authors including Duran [2], Lorentz [5], King [6], Nanda[12], [9] and Savas [17] have studied almost convergent sequences.

Let $\sigma$ be a one-to-one mapping of the set of positive integers into itself. A continuous linear functional $\varphi$ on $l_{\infty}$ is said to be an invariant mean or a $\sigma$-mean if and only if

1. $\varphi \geq 0$ when the sequence $x=\left(x_{n}\right)$ has $x_{n} \geq 0$ for all $n$.

2. $\varphi(e)=1$, where $e=(1,1, \ldots)$ and

3. $\varphi\left(x_{\sigma(n)}\right)=\varphi(x)$ for all $x \in l_{\infty}$.

For a certain kinds of mapping $\sigma$ every invariant mean $\varphi$ extends the limit functional on space $c$, in the sense that $\varphi(x)=\lim x$ for all $x \in c$. Consequently, $c \subset V_{\sigma}$ where $V_{\sigma}$ is the bounded sequences all of whose $\sigma$-means are equal, ( see, [19]).

If $x=\left(x_{k}\right)$, set $T x=\left(T x_{k}\right)=\left(x_{\sigma(k)}\right)$ it can be shown that (see, Schaefer [19]) that

$V_{\sigma}=\left\{x \in l_{\infty}: \lim _{k} t_{k m}(x)=\right.$ Le uniformly in $m$ for some $\left.L=\sigma-\lim x\right\}$

where

$t_{k m}(x)=\frac{x_{m}+T x_{m}+\ldots+T^{k} x_{m}}{k+1}$ and $t_{-1, m}=0$.

We say that a bounded sequence $x=\left(x_{k}\right)$ is $\sigma$-convergent if and only if $x \in V_{\sigma}$ such that $\sigma^{k}(n) \neq n$ for all $n \geq 0, k \geq 1$.

Just as the concept of almost convergence lead naturally to the concept of strong almost convergence, $\sigma$-convergence leads naturally to the concept of strong $\sigma$-convergence. A sequence $x=\left(x_{k}\right)$ is said to be strongly $\sigma$-convergent (see Mursaleen [10]) if there exists a number $L$ such that 
$\frac{1}{k} \sum_{i=1}^{k}\left|x_{\sigma^{i}(m)}-L\right| \rightarrow 0$

as $k \rightarrow \infty$ uniformly in $m$. We write $\left[V_{\sigma}\right]$ as the set of all strong $\sigma$ - convergent sequences. When (1.2) holds we write $\left[V_{\sigma}\right]-\lim x=\ell$. Taking $\sigma(m)=m+1$, we obtain $\left[V_{\sigma}\right]=[\hat{c}]$ so strong $\sigma$-convergence generalizes the concept of strong almost convergence.

Note that

$\left[V_{\sigma}\right] \subset V_{\sigma} \subset l_{\infty}$.

$\sigma$-convergent sequences are studied by Savas ( [13]-[16]) and others.

The summability methods of real or complex sequences by infinite matrices are of three types [see, Maddox [7], p.185] ordinary, absolute and strong. In the same vein, it is expected that the concept of invariant convergence must give rise to three types of summability methods-invariant, absolutely invariant and strongly invariant. The invariant summable sequences have been discussed by Schafer [19] and some others. More recently Mursaleen [11] have considered absolute invariant convergent and absolute invariant summable sequences. Also the strongly invariant summable sequences was studied by Saraswat and Gupta[18]. The strongly summable sequences have been systematically investigated by Hamilton and Hill [3], Kuttner [4] and some others. The spaces of strongly summable sequences were introduced and studied by Maddox [7, 8]. It is naturel to ask that how we can define a new sequence spaces by using $(\lambda, \sigma)$ - summable sequences. In this paper, we will give answer of this question and study the spaces of strongly $(\lambda, \sigma)-$ summable sequences, which naturally come up for investigation and which will fill up a gap in the existing literature.

Let $\lambda=\left(\lambda_{n}\right)$ be a non-decreasing sequence of positive numbers tending to $\infty$ such that

$$
\lambda_{n+1} \leq \lambda_{n}+1, \lambda_{1}=1
$$

The generalized de la Valèe-Poussin mean of a sequence $x$ is defined by

$$
t_{n}(x)=\frac{1}{\lambda_{n}} \sum_{k \in I_{n}} x_{k}
$$

where $I_{n}=\left[n-\lambda_{n}+1, n\right]$, for $n=1,2, \ldots$. A sequence $x=\left(x_{k}\right)$ is said to be $(V, \lambda)$ -summable to a number $L$, if $t_{n}(x) \rightarrow L$ as $n \rightarrow \infty$.

Let $A=\left(a_{n k}\right)$ be an infinite matrix of nonnegative real numbers and $p=\left(p_{k}\right)$ be a sequence such that $p_{k}>0$. (These assumptions are made throughout.) We write $A x=$ 
SAVAŞE.

$\left\{A_{n}(x)\right\}$ if $A_{n}(x)=\sum_{k} a_{n k}\left|x_{k}\right|^{p_{k}}$ converges for each $n$. We write

$$
d_{m n}(x)=\frac{1}{\lambda_{m}} \sum_{i \in I_{m}} A_{\sigma^{n}(i)}(x)=\sum_{k} a(n, k, m)\left|x_{k}\right|^{p_{k}}
$$

where

$$
a(n, k, m)=\frac{1}{\lambda_{m}} \sum_{i \in I_{m}} a_{\sigma_{n}(i), k}
$$

If $\lambda_{m}=m, m=1,2,3, \ldots$

$$
d_{m n}(x)=\frac{1}{\lambda_{m}} \sum_{i \in I_{m}} A_{\sigma_{n}(i)}(x)=\sum_{k} a(n, k, m)\left|x_{k}\right|^{p_{k}}
$$

and

$$
a(n, k, m)=\frac{1}{\lambda_{m}} \sum_{i \in I_{m}} a_{\sigma^{n}(i)}
$$

reduces to

$$
t_{m n}(x)=\frac{1}{m+1} \sum_{i=0}^{m} A_{\sigma^{n}(i)}(x)=\sum_{k} a(n, k, m)\left|x_{k}\right|^{p_{k}}
$$

where

$$
a(n, k, m)=\frac{1}{m+1} \sum_{i=0}^{m} a_{\sigma^{n}(i), k} .
$$

We now define

$$
\begin{aligned}
& {\left[A_{(\lambda, \sigma)}, p\right]_{0}=\left\{x: d_{m n}(x) \rightarrow 0 \text { uniformly in } n\right\} ;} \\
& {\left[A_{(\lambda, \sigma)}, p\right]=\left\{x: d_{m n}(x-l e) \rightarrow 0 \text { for some } l \text { uniformly in } n\right\}}
\end{aligned}
$$

and

$$
\left[A_{(\lambda, \sigma)}, p\right]_{\infty}=\left\{x: \sup _{n} t_{m n}(x)<\infty\right\}
$$

157 
The sets $\left[A_{(\lambda, \sigma)}, p\right]_{0},\left[A_{(\lambda, \sigma)}, p\right]$ and $\left[A_{(\lambda, \sigma)}, p\right]_{\infty}$ will be respectively called the spaces of strongly $(\lambda, \sigma)$-summable to zero, strongly $(\lambda, \sigma)$-summable and strongly $(\lambda, \sigma)$ bounded sequences. If $\lambda_{m}=m, m=1,2,3, \ldots$, the above spaces reduces to the following sequence spaces.

$$
\begin{aligned}
& {\left[A_{\sigma}, p\right]_{0}=\left\{x: t_{m n}(x) \rightarrow 0 \text { uniformly in } n\right\}} \\
& {\left[A_{\sigma}, p\right]=\left\{x: t_{m n}(x-l e) \rightarrow 0 \text { for some } l \text { uniformly in } n\right\}}
\end{aligned}
$$

and

$$
\left[A_{\sigma}, p\right]_{\infty}=\left\{x: \sup _{n} t_{m n}(x)<\infty\right\} \text {. }
$$

If $x$ is strongly $(\lambda, \sigma)$ - summable to $l$ we write $x_{k} \rightarrow l\left[A_{(\lambda, \sigma)}, p\right]$. A pair $(A, p)$ will be called strongly $\lambda$ - invariant regular if

$$
x_{k} \rightarrow l \Rightarrow x_{k} \rightarrow l\left[A_{(\lambda, \sigma)}, p\right]
$$

In the next Theorem, we have suitable conditions for the above sets to be complete linear topological spaces.

\section{The main results}

We first establish a number of useful propositions.

Proposition 2.1 If $p \in \ell_{\infty}$, then $\left[A_{(\lambda, \sigma)}, p\right]_{0},\left[A_{(\lambda, \sigma)}, p\right]$ and $\left[A_{(\lambda, \sigma)}, p\right]_{\infty}$ are linear spaces over $\mathbb{C}$.

Proof. We consider only $\left[A_{(\lambda, \sigma)}, p\right]$. If $H=\sup p_{k}$ and $K=\max \left(1,2^{H-1}\right)$, we have [see, Maddox [6, p. 346].

$\left|a_{k}+b_{k}\right|^{p_{k}} \leqq K\left(\left|a_{k}\right|^{p k}+\left|b_{k}\right|^{p k}\right)$

and for $\lambda \in \mathbb{C}$,

$|\lambda|^{p_{k}} \leqq \max \left(1,|\lambda|^{H}\right)$

Suppose that $x_{k} \rightarrow l\left[A_{(\lambda, \sigma)}, p\right], y_{k} \rightarrow l\left[A_{(\lambda, \sigma)}, p\right]$ and $\lambda, \mu \in \mathbb{C}$. Then we have $d_{m n}\left(\lambda x+\mu y-(\lambda l+\mu l)^{\prime} e\right) \leqq K K_{1} d_{m n}(x-l e)+K K_{2} d_{m n}\left(y-l e^{\prime}\right)$ 
where $\quad K_{1}=\sup |\lambda|^{p_{k}} \quad$ and $\quad K_{2}=\sup |\mu|^{p_{k}} \quad$, and this implies that $\lambda x+\mu y \rightarrow(\lambda l+\mu l)\left[A_{(\lambda, \sigma)}, p\right]$. This completes the proof.

We have

Proposition 2.2 $\left[A_{(\lambda, \sigma)}, p\right] \subset\left[A_{(\lambda, \sigma)}, p\right]_{\infty}$, if

$$
\|A\|=\sup _{m} \sum_{k} a(n, k, m)<\infty .
$$

Proof. Assume that $x_{k} \rightarrow l\left[A_{(\lambda, \sigma)}, p\right]$ and (2.3) holds. Now by the inequality (2.1),

$$
\begin{aligned}
d_{m n}(x) & =t_{m n}(x-l e+l e)(4) \\
\leqq & K d_{m n}(x-l e)+K \sum_{k} a(n, k, m)|l|^{p_{k}} \\
\leqq & K d_{m n}(x-l e)+K\left(\sup |l|^{p_{k}}\right) \sum_{k} a(n, k, m) .
\end{aligned}
$$

Therefore $x \in\left[A_{(\lambda, \sigma)}, p\right]_{\infty}$ and this completes the proof.

Remark 2.3 Some known sequence spaces are obtained by specializing $A$ and therefore some of the results proved here extend the corresponding results obtained for the special cases.

Proposition 2.4 Let $p \in \ell_{\infty}$ then $\left[A_{(\lambda, \sigma)}, p\right]_{0}$ and $\left[A_{(\lambda, \sigma)}, p\right]_{\infty}\left(\inf p_{k}>0\right)$ are linear topological spaces paranormed by $g$ defined by

$$
g(x)=\sup _{m, n}\left[d_{m, n}(x)\right]^{1 / M}
$$

where $M=\max \left(1, H=\sup p_{k}\right)$. If $(2.3)$ holds, then $\left[A_{\lambda}, p\right]$ has the same paranorm.

Proof. Clearly $g(0)=0$ and $g(x)=g(-x)$. Since $M \geqq 1$, by Minkowski's inequality it follows that $g$ is subadditive. We now show that the scalar multiplication is continuous. It follows from the inequality (2.2) that

$g(\lambda x) \leqq \sup |\lambda|^{p_{k}^{\prime M}} g(x)$

Therefore $x \rightarrow 0 \Rightarrow \lambda x \rightarrow 0$ (for fixed $\lambda$ ). Now let $\lambda \rightarrow 0$ and $x$ be fixed. Given $\varepsilon>0 \quad \exists N$ such that 
$d_{m, n}(\lambda x)<\varepsilon / 2(\forall n, \forall m>N)$.

Since $d_{m, n}(x)$ exists for all $m$, we write

$d_{m, n}(x)=K(m),(1 \leqq m \leqq N)$

and

$\delta=\left(\frac{\varepsilon}{2 K(m)}\right)^{1 / p_{k}}$.

Then $|\lambda|<\delta$,

$d_{m, n}(\lambda x)<\frac{\varepsilon}{2}(\forall n, 1 \leqq m \leqq N)$.

It follows from (2.5) and (2.6) that

$\lambda \rightarrow 0 \Rightarrow \lambda x \rightarrow 0(x$ fixed $)$

This proves the assertion about $\left[A_{(\lambda, \sigma)}, p\right]_{0}$. If inf $p_{k}=\theta>0$ and $0<|\lambda|<1$, then $\forall x \in\left[A_{(\lambda, \sigma)}, p\right]_{\infty}$,

$g^{M}(\lambda x) \leqq|\lambda|^{\theta} g^{M}(x)$

Therefore $\left[A_{\lambda}, p\right]_{\infty}$ has the paranorm $g$. If (2.3) holds it is clear from Proposition 2.2 that $g(x)$ exists for each $x \in\left[A_{(\lambda, \sigma)}, p\right]$. This completes the proof.

Remark 2.5 It is evident that $g$ is not a norm in general. But if $p_{k}=p \quad \forall k$, then clearly $g$ is a norm for $1 \leqq p \leqq \infty$ and a $p$ - norm for $0<p<1$.

Proposition 2.6 $\left[A_{\lambda}, p\right]_{0}$ and $\left[A_{(\lambda, \sigma)}, p\right]_{\infty}$ are complete with respect to their paranorm topologies $\left[A_{(\lambda, \sigma)}, p\right]$ is complete if (2.3) holds and

$\sum_{k} a(n, k, m) \rightarrow 0$ uniformly in $n$.

Proof. Let $\left\{x^{i}\right\}$ be a Cauchy sequence in $\left[A_{(\lambda, \sigma)}, p\right]_{0}$. Then there exists a sequence $x$ such that $g\left(x^{i}-x\right) \rightarrow 0 \quad(i \rightarrow \infty)$. Since $g$ is subadditive it follows that $x \in\left[A_{\lambda}, p\right]_{0}$. The 
completness of $\left[A_{(\lambda, \sigma)}, p\right]_{\infty}$ can be similarly obtained. We now consider $\left[A_{(\lambda, \sigma)}, p\right]$. If (2.3) holds and $\left\{x^{i}\right\}$ is a Cauchy sequence in $\left[A_{\lambda}, p\right]$, Then there exists $x$ such that $g\left(x^{i}-x\right) \rightarrow 0$. If (2.7) holds then from inequality (2.4) it is clear that $\left[A_{(\lambda, \sigma)}, p\right]=\left[A_{(\lambda, \sigma)}, p\right]_{0}$. This completes the proof.

Combining the above facts we obtain the main result.

Theorem 2.7 Let $p \in \ell_{\infty}$. Then $\left[A_{(\lambda, \sigma)}, p\right]_{0}$ and $\left[A_{(\lambda, \sigma)}, p\right]_{\infty}\left(\right.$ inf $\left.p_{k}>0\right)$ are complete linear topological spaces paranormed by $g$. If (2.3) and (2.7) hold then $\left[A_{(\lambda, \sigma)}, p\right]$ has the same property. If further $p_{k}=p$ for all $k$, they are Banach spaces for $1 \leqq p<\infty$ and $p$-normed spaces for $0<p<1$.

\section{References}

[1] Banach, S., Theorie des Operations Lineaires, (1932).

[2] Duran, J.P., Infinite matrices and almost convergence, Math. Z., 128, 75-83, (1972).

[3] Hamilton, H.J. and Hill, J. D., On strong summability, Amer. J. Math., 60, 588-94, (1938).

[4] Kuttner, B., Note on strong summability, J. London Math. Soc., 21, 118-22, (1946).

[5] King, J.P., Almost summable sequences, Proc. Amer. Math. Soc., 17, 1219-25, (1966).

[6] Lorentz, G.G., A contribution to the theory of divergent sequences, Acta Math., 80, 167-190, (1948).

[7] Maddox, I.J., Spaces of strongly summable sequences, Quart. J. Math. Oxford Ser., (2)18, 345-55, (1967).

[8] Maddox, I.J., Elements of Functional Analysis, Cambridge University Press, (1970).

[9] Malkowsky, E. and Savaş, E., Some $\lambda$-sequence spaces defined by a modulus, Archivum Math., 36(3), 219-228, (2000).

[10] Mursaleen, M., Matrix transformation between some new sequence spaces, Houston J. Math., 9, 505-509, (1993),.

[11] Mursaleen, M., On some new invariant matrix methods of summability, Q.J. Math., 34, 77-86, (1983).

[12] Nanda, S., Some sequence spaces and almost convergence, J. Austral. Math. Soc. (Series A), 22, 446-455, (1976).

[13] Savaş, E., Some sequence spaces involving invariant means, Indian J. Math., 31, (1989).

[14] Savaş, E., A note on some sequence spaces, Doğa Türk. J. Math., 15, (1991).

[15] Savaş, E., Invariant means and generalization of a theorem of S. Mishra, Doga Türk. J. Math., 14, (1989).

[16] Savaş, E., Invariant coregular and conull matrices of operators, Hacettepe Bull. Math. Sci. and Eng., 19, (1990).

[17] Savaş, E., Infinite matrices and generalized almost convergence, Doga Türk. J. 
Math., 5(3), 1-10, (1987).

[18] Saraswat, S.K. and Gupta, S.K., Spaces of strongly $\sigma$-summable sequences, Bull. Cal. Math. Soc., 75, 179-184, (1983).

[19] Schaefer, P., Infinite matrices and invariant means, Proc. Amer. Math. Soc., 36, 104-110, (1972). 